\title{
El contrato psicológico, el desempeño y la satisfacción en empleados de organizaciones públicas y privadas de Lima
}

\author{
The psychological contract, performance and satisfaction of employees of \\ public and private organizations of Lima
}

\author{
Alejandro Loli ${ }^{1 *}$, Vladimir Navarro ${ }^{2}$
}

Universidad Nacional Mayor de San Marcos

Javier Del Carpio ${ }^{3}$

Universidad ESAN

\section{Diana Castillo ${ }^{5}$, Alejandro Espinoza ${ }^{6}$, Ofelia Borja ${ }^{7}$ Universidad Nacional Mayor de San Marcos}

Recibido: $08-05-17$

\author{
Arturo Vergara ${ }^{4}$ \\ Universidad Nacional Agraria La Molina
}

\section{Resumen}

Teóricos e investigadores han coincidido en señalar que el contrato psicológico es un proceso que hace referencia a obligaciones recíprocas e implícitas entre el empleado y el empleador, y que va más allá de los contratos explícitos y/o formales. Esta situación que antes de la década de 1990 se mantenía estable, adquiere importancia en los tiempos actuales, debido a cambios bruscos en la modalidad de los empleos en las organizaciones actuales. De manera que, el objeto del presente estudio es conocer el estado actual del contrato psicológico y su relación con el desempeño y la satisfacción en 303 empleados del sector público y privado de Lima. Los resultados nos indican que existe una correlación significativa y positiva entre el contrato psicológico, el desempeño y la satisfacción. Entre tanto, existen diferencias entre las obligaciones de la organización según sea pública o privada, impactando más en las organizaciones privadas; ocurriendo de la misma manera con la satisfacción laboral. Similares diferencias, se encontró entre las variables de estudio según el género, estado civil, estatus ocupacional, remuneraciones y lugar de procedencia; no así con la edad, años de servicio en el puesto y antigüedad en la organización.

Palabras clave: Contrato psicológico, desempeño, satisfacción, empleados públicos y privados, Lima, Perú.

\footnotetext{
*Autor para correspondencia

1. Universidad Nacional Mayor de San Marcos. Email: alolip@unmsm.edu.pe

2. Universidad Nacional Mayor de San Marcos. Email: vnavarrov@unmsm.edu.pe

3. Universidad ESAN. Email jdelcarpio1@yahoo.es

4. Universidad Nacional Agraria La Molina Email: mineriadifusa@gmail.com

5. Universidad Nacional Mayor de San Marcos Email: dianacastillocanales@gmail.com

6. Universidad Nacional Mayor de San Marcos Email: alejandromagno765@hotmail.com

7. Universidad Nacional Mayor de San Marcos Email: ofelia.borja@unmsm,edu,pe
} 


\begin{abstract}
Theorists and researchers have agreed that the psychological contract is a process that refers to reciprocal and implicit obligations between the employee and the employer, and goes beyond explicit and / or formal contracts. This situation, which remained stable before the 1990 s, is becoming more important in the present times, due to sudden changes in the modality of jobs in the current organizations. For that reason, the objective of the present study is to know the current state of the psychological contract and its relationship with performance and satisfaction in 303 employees of the public and private sector of Lima. The results show that there is a significant and positive correlation between psychological contract, performance and satisfaction. In the meantime, there are differences between the obligations of the organization according to whether public or private, impacting more on private organizations; happening in the same way with job satisfaction. Similar differences were found among study variables according to gender, marital status, occupational status, wages and place of residence; however the results are different when variables such as age, years of service in the position and seniority in the organization are analyzed
\end{abstract}

Keywords: psychological contract, performance, satisfaction, public and private employees, Lima, Peru.

El actual modelo económico ha generado cambios profundos en la relación organización-trabajador y en las formas de conducción de dichas relaciones. Aquello que surgió como una percepción laboral de ambas partes o como obligaciones implícitas de la relación como producto de la cultura organizacional informal y como una adaptación a un sistema disfuncional (Argyris, 1960; en Tena, 2002), o un conjunto de expectativas no escritas e implícitas que operan en todo momento entre cada miembro de una organización y otros miembros de esa misma organización, creando una interdependencia entre trabajador y organización que beneficia a ambas partes al incrementar la productividad, la eficiencia organizacional, la confianza y la satisfacción laboral (Levinson, 1962; Schein, 1965), se llama contrato psicológico; y, hoy en día, está siendo observado después de muchos años de vigencia.

Así, la posición de Levinson, Schein \& Argyris, respecto del contrato psicológico tiene muchos cuestionamientos (Tena, 2002), probablemente, uno de los más importantes es la de Rousseau (1989), cuando señala que se "necesita una visión más realista de los contratos debido a las cambiantes necesidades laborales", atribuyéndoles a los puntos de vista de los autores primigenios, connotaciones puramente económicas (contrato transaccional), dejando de lado aspectos socioemocionales (contrato relacional), de vital importancia en la relación laboral empleador-empleado (Tena, 2002).

Estas creencias y expectativas de las partes, que se debe más a percepciones individuales que organizacionales, dicen mucho sobre la actuación de quienes establecen los primeros contactos a nombre de la organización con las personas que desean incorporarse a la misma y quienes se incorporan como consecuencia de un proceso previo de reclutamiento y selección; incluso antes, cuando logran tener un concepto sobre la base de la imagen que trasciende la organización hacia su entorno social (Saldarriaga, 2013), permitiéndole al interesado tomar decisiones 
sobre sus expectativas ya formadas, acudiendo al proceso de selección de personal y confirmando, muchas veces, sus expectativas en el contacto directo (Dunahee \& Wangler, 1974). Vale decir, los encargados de la selección de personal o los primeros contactos con los supervisores que se atribuyen la representación de la organización, crea expectativas, o los postulantes que perciben tal función cuando tal vez no existe intención alguna. De este modo, se podría decir que, la organización como tal no interviene directamente en este proceso. En todo caso cada quien crea sus propias expectativas respecto del otro.

Sobre las expectativas, De Luca (2012), señala que existen estudios que indican un conjunto de aspectos de los que estaría conformado el contenido de las expectativas de los empleados hacia la organización, entre ellas, el salario, mejora de ingresos, posibilidad de crecimiento dentro de la organización, buena relación con el jefe directo, cooperación grupal, buen clima laboral, tiempo libre para la vida personal, trabajo desafiante, estimulante y creativo, entrenamiento y educación, libertad para tomar decisiones, ser consultado por el superior, comunicación abierta y directa, trato honesto y justo, y que la organización valore el trabajo. Y, de parte de los empleadores hacia los empleados, que den una buena imagen de la empresa, lealtad, motivación, esfuerzo y disposición al sacrificio, trabajar bien en equipo, trabajar horas extras si se requiere para llegar a un objetivo, entregar un buen trabajo en orden de cantidad y calidad, y aceptar una transferencia interna si fuera necesario (Schein, 1982).

Independientemente a esta discusión, aquellas concepciones comienzan perder vigencia debido a los nuevos escenarios en las relaciones laborales entre los empleadores y los trabajadores, donde el empleador contrata a un trabajador para laborar en otra empresa (tercerización), los contratos se dan a tiempo definido (temporales) y, por lo mismo, se producen desplazamientos de trabajadores permanentemente, de manera que el trabajador pierde identidad, compromiso organizacional, satisfacción laboral y confianza; vale decir, que la ruptura tiene consecuencias negativas (Topa y Palací, 2004), consecuentemente el desempeño y la satisfacción no son los mismos (Topa, Lisbona, Palaci y Morales 2002; Topa y Palací, 2004) y el contrato psicológico se debilita y demanda de un nuevo constructo, aunque existen trabajadores que sienten preferencia por este tipo de contratos poco estables (Barringer \& Milkovich, 2002, en Blanch, [2009), pero también las hay de aquellos que creen en la adecuación del contrato laboral a sus necesidades (Solanes y Zaragoza, 2010).

De ese modo, el contrato psicológico se convierte en un tema de interés para entender el comportamiento de los trabajadores pero también el de la organización; además, el contrato psicológico se ha configurado en un concepto clave de las nuevas relaciones laborales (Gracia, Silla, Peiró y Fortes-Ferreira, 2006) de hecho, estos mismos autores encuentran que el cumplimiento del contrato psicológico correlaciona positivamente con el bienestar psicológico, la satisfacción con la vida 
y con el trabajo, compromiso organizacional, evaluación positiva de las relaciones de empleo; y negativamente con propensión al abandono y conflicto trabajo/familia; en el mismo sentido Topa, y Morales (2007), encuentran que la ruptura del contrato psicológico está asociada a un incremento de resultados indeseables, descenso de los deseables y del compromiso organizacional, también a un incremento en la intención de abandono y negligencia en las tareas. Por ello, incluso existen autores que señalan que el contrato psicológico, visto así, demanda de un replanteamiento debido a los cambios en la manera de conducir la organización y las diversas formas de empleo, donde el contrato psicológico debe verse como una relación de lealtad del trabajador vs potenciación de la persona para la empleabilidad en lugar de estabilidad (Calderón, Naranjo y Álvarez, 2007).

Este nuevo planteamiento en la relación laboral, aún en discusión, permite conocer las exigencias de lealtad que las organizaciones esperan de los trabajadores y, en compensación, ofrecer mejoría de sus capacidades (conocimientos, destrezas y desarrollo personal); es decir, potenciar las competencias de los trabajadores para que puedan encontrar con facilidad otros empleos en el mercado laboral. Sin embargo, los empleadores, en la práctica, asumen sus propias estrategias al actuar de manera evasiva mediante la contratación de personal con competencias "múltiples", con el objeto de cubrir no sólo las tareas de un puesto de trabajo, si no realizar muchas actividades de diversos puestos de trabajo. La razón sustancial sería bajar sus costos de producción, aún con el riesgo de no lograr su lealtad o perder su permanencia en la organización. De hecho, aquí, las expectativas de la organización, expresadas en políticas explícitas o implícitas, si bien distintas y ambiciosas, son más expresivas que las del empleado que se incorpora.

Si bien es cierto que, la expectativa hoy, es buscar personal útil para todas las actividades, aún en estas condiciones, ninguna de las partes puede evitar la presencia de las creencias y expectativas que se dan entre ellas. Por consiguiente, siempre habrá compromisos y obligaciones mutuas e implícitas, aún en la nueva situación del contrato psicológico, que de no cumplirse -como se dijo antes- pueden generar un conjunto de consecuencias imprevisibles, pero el más inmediato será la frustración y la ansiedad (Morrison y Robinson, 1997). Las reacciones que resulten de ella, dependerá de las personas y pueden ir desde agresiones indirectas como las de rescindir su compromiso de lealtad, apatía hacia el trabajo y hacia la empresa, limitarse a cumplir las tareas de su puesto y cumplir su horario (Huse $\&$ Bowdith, 1988), conduciendo a un descenso de la confianza, disminución de su alto desempeño, merma en la satisfacción organizacional (Robbins, 1994), afectando la cantidad y calidad de la producción; o agresiones directas y visibles, como la de organizar un sindicato para hacer causa común en sus necesidades, sus frustraciones y sus logros y/u optar por no permanecer un día más frente a la ruptura del contrato psicológico, hasta provocar su despido intempestivo a fin de sacar alguna ventaja económica. Es decir, la decepción y el resentimiento, estaría 
conduciendo a una relación laboral más transaccional que relacional (Tena, 2002) o la ruptura laboral definitiva.

Como se aprecia, los nuevos escenarios organizacionales cambian la orientación del contrato psicológico, rompe con la tradicional estabilidad laboral, donde el empleador ofrecía seguridad en el trabajo, por tanto, podría mantenerse por muchos años, retirarse solo al cumplir su vida útil, eventualmente, ascender y promoverse a puestos más expectantes y crecer como persona y tal vez mejorar económicamente que beneficiaría a toda su familia; y donde el trabajador ofrecía su lealtad, y la obediencia para el cumplimiento de los objetivos y necesidades de la organización. Ahora, el nuevo contrato psicológico, prevé una alta dosis de lealtad del empleado hacia su empleador, dependerá de esa lealtad para lograr algunos beneficios o el éxito económico. En este escenario, el crecimiento personal está sujeta a los sacrificios individuales de los empleados, éstos "deben buscar nuevas vías para aumentar sus competencias; las oportunidades de promoción serán limitadas y la movilidad será más horizontal que vertical; la flexibilidad y las competencias generales facilitarán la empleabilidad, porque los empleados más deseados en estas nuevas organizaciones serán aquellos que puedan trabajar en una variedad de entornos, se puedan mover fácilmente a través de las barreras funcionales y sepan cómo utilizar diferentes técnicas" (Tena, 2002). Aun cuando se espera que las mejoras en las potencialidades de los empleados, debe estar a cargo del empleador, por ejemplo, en la forma de pasantías en el caso de jóvenes que se inician en la vida laboral (Hurst, Good \& Gardner, 2012). No hay ninguna seguridad que eso sea así. Tendrá que ganársela. Las consecuencias para el empleador pueden ser muy duras, pero los riesgos están planteados. Como decían Gallie \& White (1993) "este cambio conlleva indudablemente determinados riesgos, como son las probables repercusiones motivacionales y actitudinales en el trabajador", por tanto, las creencias y expectativas están claras y se mantendrán inalterables, en tanto exista una relación laboral, aun cuando la voluntad por alterarlos sea una realidad de parte.

Por las razones señaladas, es preocupación de este estudio, conocer el estado actual del contrato psicológico y su relación con la satisfacción y el desempeño en 303 empleados del sector público y privado de Lima.

\section{MÉTODO}

\section{Muestra}

La muestra fue intencional y no probabilístico y su composición fue de 303 empleados, profesionales de diversas especialidades que venían cursando maestrías con mención en negocios internacionales, gestión pública, gestión empresarial, administración, psicología clínica, psicología educativa, psicología organizacional, y doctorados en varias disciplinas; además, varios diplomados y segunda especialización en las Facultades de Administración y Psicología 
respectivamente. Todos ellos, empleados o funcionarios de organizaciones públicas y privadas de Lima, Perú.

\section{Instrumentos}

\section{Cuestionario de contrato psicológico empleador / trabajador}

Se utilizó el cuestionario de Rigotti, Mohr, De Cuyper \& De Witte (2003) para medir el contrato psicológico empleador/trabajador, desde la percepción del trabajador, en los dos sentidos: las obligaciones de su organización (empleador) y sus compromisos con la organización (empleado). Este cuestionario en su componente de obligaciones de la organización está compuesto por 15 ítemes y en su componente de compromisos del trabajador está constituido por 17 ítemes, ambos con seis (6) alternativas de repuestas por ítem, que van desde "No ha cumplido" (0), hasta "Si, y ha cumplido su promesa por completo" (5). Ambas dimensiones, fueron sometidas a análisis factorial mediante el Coeficiente Alpha De Crombach, obteniendo un nivel de confiabilidad de 0.94 y 0.91 respectivamente y una alta consistencia interna ( $a=.94$ y $a=.91)$. Lo que nos muestra que el instrumento tiene estabilidad.

\section{Cuestionario de satisfacción laboral}

El cuestionario utilizado pertenece a Aguirre y Vauro (2007), está compuesto por 12 itemes con seis alternativas de respuesta que van de "Muy insatisfecho (1) a Muy satisfecho (6)". Sometido a análisis factorial mediante el coeficiente alpha de Crombach, su nivel de confiabilidad fue de 0.91, lo que significa que la prueba es estable y confiable, confirmándose con una alta consistencia interna $(a=.91)$.

Tabla 1

Composición de la muestra de estudio

\begin{tabular}{llcc}
\hline Variable socio demográfica & $\mathrm{n}$ & $\%$ \\
\hline \multirow{2}{*}{ Sexo } & Mujer & 193 & 63.7 \\
& Varón & 110 & 36.3 \\
& 18 a 30 años & 103 & 34.0 \\
Edad & 31 a 40 años & 129 & 42.6 \\
& 41 a 50 años & 48 & 15.8 \\
& 51 años a más & 23 & 7.6 \\
Estado & Soltero & 183 & 60.4 \\
civil & Casado & 101 & 33.3 \\
& Divorciado & 14 & 4.6 \\
\multirow{2}{*}{ Grado de } & Unión libre & 5 & 1.7 \\
instrucción & Universitario & 154 & 50.8 \\
Total & Maestría & 148 & 48.8 \\
\hline
\end{tabular}




\section{Cuestionario de desempeño laboral}

La prueba tiene como autor a Abramis (1994), y está compuesto por seis ítemes con seis alternativas de respuesta, va desde "Muy mal (1) hasta Muy bien (6)". Sometido a análisis factorial mediante el coeficiente alpha de Crombach, el nivel de confiabilidad que le da estabilidad al cuestionario fue de 0.87 , con un buen nivel de consistencia interna $(a=.87)$.

Tabla 2

Confiabilidad alfa de Cronbach de cada una de las pruebas

\begin{tabular}{lccccc}
\hline Variable & $\boldsymbol{M}$ & $\boldsymbol{D E}$ & $\begin{array}{c}\text { Alfa de } \\
\text { Cronbach }\end{array}$ & $\mathbf{I C ~ 9 5 \%}$ & $\mathbf{N}^{\mathbf{0}}$ de ítems \\
\hline Obligaciones & 48.41 & 14.21 & .94 & {$[.93,95]$} & 15 \\
Compromiso & 69.42 & 10.23 & .91 & {$[.90, .92]$} & 17 \\
Satisfacción & 51.89 & 9.80 & .91 & {$[.90, .93]$} & 12 \\
Desempeño & 29.29 & 3.71 & .87 & {$[.85, .89]$} & 6 \\
\hline
\end{tabular}

\section{Procedimiento}

Los tres cuestionarios fueron aplicados en las aulas de clase en forma colectiva por los autores del presente artículo con el apoyo de jóvenes estudiantes de psicología de los últimos ciclos de estudio, con experiencia en la aplicación de pruebas psicológicas, $\mathrm{y}$ entrenados previamente para este fin.

\section{Procesamiento de datos}

Se utilizó el paquete estadístico SPSS (Statistical Package for the Social Sciences) para el tratamiento estadístico. De manera especial se utilizó el coeficente Alpha de Crombach para determinar los grados de confiabilidad, medidas de tendencia central para ver los grados de cumplimiento de obligaciones del empleador y los compromisos del empleado para con su organización, el estadístico de correlación de Rho Spearman para establecer la asociación entre los diferentes factores, el puntaje "t" y el puntaje "z", el Chi Cuadrado y otros para ver la diferencia entre los grupos en las variables socio demográficas.

\section{RESULTADOS}

\section{Niveles de cumplimiento de obligaciones de la organización en el contrato psicológico}

En términos generales, se puede decir que el nivel de cumplimiento de obligaciones de la organización respecto del contrato psicológico, en la percepción de la mayoría de los empleados de las organizaciones públicas y privadas de Lima, es que cree que está en el nivel medio (47.9\%), casi un tercio (27.0\%) de los mismos dice que es alto y el $25.1 \%$ cree que el nivel de cumplimiento de obligaciones es bajo. 


\section{Tabla 3}

Niveles de cumplimiento de obligaciones

\begin{tabular}{lcc}
\hline Nivel & Frecuencia & $\begin{array}{c}\text { Porcentaje } \\
\text { válido }\end{array}$ \\
\hline Bajo & 76 & 25.1 \\
Medio & 145 & 47.9 \\
Alto & 82 & 27.0 \\
Total & 303 & 100 \\
\hline
\end{tabular}

\section{Niveles de compromiso de los empleados con su organización}

Igualmente, el nivel de compromiso de los empleados con su organización, respecto del contrato psicológico, la mayoría de empleados profesionales en las organizaciones públicas y privadas de Lima percibe que es de nivel medio (45.2\%), mientras que casi un tercio de ellos percibe que es bajo (27.7\%), y el otro tercio de ellos percibe que tiene un nivel de compromiso alto para con su organización (27.1\%).

\section{Tabla 4}

Nivel de compromiso de los empleados con su organización.

\begin{tabular}{lcc}
\hline Nivel & Frecuencia & $\begin{array}{c}\text { Porcentaje } \\
\text { válido }\end{array}$ \\
\hline Bajo & 84 & 27.7 \\
Medio & 137 & 45.2 \\
Alto & 82 & 27.1 \\
Total & 303 & 100 \\
\hline
\end{tabular}

\section{Niveles de satisfacción laboral de los empleados}

El nivel de satisfacción laboral predominante de los empleados, se podría decir que es medio (46.9\%), en cambio un tercio de ellos percibe que es alto (27.4\%), y el $25.7 \%$ cree que es bajo el nivel de satisfacción.

\section{Niveles de desempeño laboral de los empleados}

En términos generales, el nivel de desempeño laboral percibido por los empleados, se podría afirmar que corresponde al nivel medio (38.0\%), más de un tercio de ellos considera que es bajo (36.6\%) y solo el $25.4 \%$ cree que es alto.

Tabla 5

Niveles de satisfacción laboral de los empleados.

\begin{tabular}{lcc}
\hline Nivel & Frecuencia & \% válido \\
\hline Bajo & 78 & 25.7 \\
Medio & 142 & 46.9 \\
Alto & 83 & 27.4 \\
Total & 303 & 100 \\
\hline
\end{tabular}


Tabla 6

Niveles de desempeño laboral de los empleados

\begin{tabular}{lcc}
\hline Nivel & Frecuencia & \% válido \\
\hline Bajo & 111 & 36.6 \\
Medio & 115 & 38.0 \\
Alto & 77 & 25.4 \\
Total & 303 & 100 \\
\hline
\end{tabular}

\section{Asociación del contrato psicológico con la satisfacción laboral y el desempeño de los empleados públicos y privados de Lima}

La tabla 7, muestra la correlación entre las obligaciones del empleador y el compromiso de los empleados para con su organización (contrato psicológico) con la satisfacción laboral y el desempeño de los empleados de las organizaciones públicas y privadas de Lima. En ella, se encontró que existe correlación significativa y positiva entre obligaciones de la organización y el compromiso de los empleados con su organización $\left(\mathrm{r}=.349^{* *}\right)$. Igualmente, hay una correlación significativa y positiva entre obligaciones de la organización y la satisfacción laboral $(\mathrm{r}=$ $\left..721^{* *}\right)$ y éste con compromiso con su organización $\left(\mathrm{r}=.437^{* *}\right)$; por otro lado, se encontró una correlación significativa y positiva de obligación de la organización con desempeño laboral $\left(\mathrm{r}=.285^{* *}\right)$ y éste con compromiso de los empleados con su organización $\left(\mathrm{r}=.568^{* *}\right)$ y con la satisfacción laboral $\left(\mathrm{r}=.376^{* *}\right)$; es decir, cuanto mayor es el cumplimiento de las obligaciones del empleador, mayor será el compromiso de los empleados con su organización, mayor la satisfacción laboral y mayor el desempeño laboral; del mismo modo, cuanto mayor es el compromiso con su organización, mayor será la satisfacción laboral y mayor el desempeño laboral de los empleados. Igual, a mayor satisfacción laboral mayor desempeño laboral. Probablemente, debido a que el contrato psicológico está directamente asociado con la satisfacción y el desempeño laboral.

\section{Tabla 7}

Correlaciones entre obligaciones de la organización, compromiso con su organización, la satisfacción laboral y el desempeño

\begin{tabular}{lcccc}
\hline & $\begin{array}{c}\text { Obligaciones de la } \\
\text { organización }\end{array}$ & $\begin{array}{c}\text { Compromiso con su } \\
\text { organización }\end{array}$ & $\begin{array}{c}\text { Satisfacción } \\
\text { laboral }\end{array}$ & $\begin{array}{c}\text { Desempeño } \\
\text { laboral }\end{array}$ \\
\hline $\begin{array}{l}\text { Compromiso con } \\
\text { su organización }\end{array}$ & - & - & & \\
$\begin{array}{l}\text { Satisfacción } \\
\text { laboral }\end{array}$ & $.349^{* *}$ & $.437^{* *}$ & - & \\
$\begin{array}{l}\text { Desempeño } \\
\text { laboral }\end{array}$ & $.721^{* *}$ & $.568^{* *}$ & $.376^{* *}$ & - \\
\hline
\end{tabular}

Rho Spearman: $* * p<.01 \mathrm{~N}=303$

** La correlación es significativa al nivel 0,01 (bilateral). 


\section{Diferencias del contrato psicológico según las variables socio demográficas}

a. Obligaciones del empleador y la satisfacción del empleado según el sexo.

En la tabla 8 se muestra, con respecto al sexo, que existe diferencias estadísticamente significativas en obligaciones de la organización $(t=-2.079$, $p<.05)$, siendo la puntuación de los varones $(\mathrm{M}=50.65, \mathrm{DE}=13.45)$ superior al de las mujeres $(\mathrm{M}=47.13, \mathrm{DE}=14.50)$ con un tamaño de efecto pequeño $\left(d_{\text {Cohen }}=.248\right)$. En cambio, se observa que no existe diferencias estadísticamente significativas en satisfacción laboral $(t=-.853, p>.05)$ con un tamaño de efecto muy bajo $\left(d_{\text {Cohen }}=.101\right)$. En otros términos, la condición del género (ser mujer o varón) hace que varíe la percepción de los empleados respecto de las obligaciones de la organización.

Tabla 8

Obligaciones del empleador y la satisfacción del empleado según el sexo

\begin{tabular}{lccccccc}
\hline Variable & Sexo & $\mathbf{n}$ & $\mathbf{M}$ & $\mathbf{D E}$ & $\boldsymbol{t}$ & $\boldsymbol{p}$ & $\mathbf{d}_{\text {Cohen }}$ \\
\hline \multirow{2}{*}{ Obligaciones } & Mujer & 193 & 47.13 & 14.50 & \multirow{2}{*}{.079} & .038 & .248 \\
& Varón & 110 & 50.65 & 13.45 & & & \\
\hline \multirow{2}{*}{ Satisfacción } & Mujer & 193 & 51.53 & 9.96 & -.853 & .395 & .101 \\
& Varón & 110 & 52.53 & 9.51 & & & \\
\hline
\end{tabular}

De la misma manera, que en la variable satisfacción laboral, no se encontraron diferencias estadísticamente significativas en compromiso de los empleados con la organización y desempeño laboral, según el sexo.

b. Obligaciones de la organización, compromiso con su organización, satisfacción y desempeño laboral, según el estado civil.

La tabla 9 nos muestra que, según el estado civil, existe diferencias estadísticamente significativas en satisfacción laboral $\left(\chi^{2}=12.455, p<.01\right)$, siendo las puntuaciones de las personas divorciadas $(\mathrm{Rp}=188.68)$, casadas $(\mathrm{Rp}=163.97)$ y solteras $(\mathrm{Rp}=145.44)$ mayores a las de unión libre $(\mathrm{Rp}=47.80)$. En cambio, esto no se observó en obligaciones de la organización $\left(\chi^{2}=4.363, p>.05\right)$, compromiso con su organización $\left(\chi^{2}=3.170, p>.05\right)$ y desempeño laboral $\left(\chi^{2}=1.383, p>.05\right)$. Vale decir, que el estado civil de los empleados influye en la percepción respecto de la satisfacción laboral.

Tabla 9

Diferencias de las obligaciones de la organización compromiso con su organización, satisfacción y desempeño, según el estado civil

\begin{tabular}{llcccc}
\hline Variable & Estado civil & N & $\begin{array}{c}\text { Rango } \\
\text { promedio }\end{array}$ & $\boldsymbol{\chi}^{2}$ & $\boldsymbol{p}$ \\
\hline \multirow{3}{*}{ Obligaciones } & Soltero & 183 & 144.74 & & \\
& Casado & 101 & 166.17 & 4.363 & .225 \\
& Divorciado & 14 & 154.04 & & \\
\hline & Unión libre & 5 & 125.60 & & \\
\hline
\end{tabular}




\begin{tabular}{llcccc}
\hline \multirow{3}{*}{ Compromiso } & Soltero & 183 & 149.59 & & \\
& Casado & 101 & 150.38 & 3.170 & .366 \\
& Divorciado & 14 & 175.50 & & \\
& Unión libre & 5 & 207.20 & & \\
& Soltero & 183 & 145.44 & & \\
Satisfacción & Casado & 101 & 163.97 & 12.455 & .006 \\
& Divorciado & 14 & 188.68 & & \\
& Unión libre & 5 & 47.80 & & \\
& Soltero & 183 & 150.03 & & .710 \\
\multirow{3}{*}{ Desempeño } & Casado & 101 & 151.89 & \multirow{2}{*}{1.383} & \\
& Divorciado & 14 & 164.79 & & \\
& Unión libre & 5 & 190.70 & & \\
\hline
\end{tabular}

c. Obligaciones de la organización, compromiso con su organización, satisfacción y desempeño laboral, según tipo de organización: pública y privada.

En la tabla 10, encontramos que existe diferencias estadísticamente significativas entre las obligaciones de la organización según el tipo de organización $(\mathrm{Z}=-2.399, p<.05)$ impactando más en las organizaciones privadas. Así mismo, existe diferencias estadísticamente significativas entre satisfacción laboral según el tipo de organización $(\mathrm{Z}=-2.467, p<.05)$, impactando más en las organizaciones privadas que públicas. Significa, que dependiendo del tipo de organización en la cual trabajan (pública o privada), la percepción de los empleados cambia respecto de las obligaciones de la organización, así como de la satisfacción laboral.

\section{Tabla 10}

Obligaciones de la organización, compromiso con su organización, satisfacción y desempeño laboral, según tipo de organización: pública y privada

\begin{tabular}{|c|c|c|c|c|c|c|c|}
\hline Variable & $\begin{array}{l}\text { Entidad y/o } \\
\text { empresa }\end{array}$ & $\mathbf{n}$ & $\begin{array}{c}\text { Rango } \\
\text { promedio }\end{array}$ & $\boldsymbol{U}$ & $Z$ & $p$ & $r_{p}$ \\
\hline \multirow{2}{*}{ Obligaciones } & Pública & 113 & 135.48 & \multirow{2}{*}{8868.5} & \multirow{2}{*}{-2.399} & \multirow{2}{*}{.016} & \multirow{2}{*}{.138} \\
\hline & Privada & 188 & 160.33 & & & & \\
\hline \multirow{2}{*}{ Compromiso } & Pública & 113 & 159.11 & \multirow{2}{*}{9705.5} & \multirow{2}{*}{-1.254} & \multirow{2}{*}{.210} & \multirow{2}{*}{.072} \\
\hline & Privada & 188 & 146.13 & & & & \\
\hline \multirow{2}{*}{ Satisfacción } & Pública & 113 & 135.05 & \multirow{2}{*}{8819.5} & \multirow{2}{*}{-2.467} & \multirow{2}{*}{.014} & \multirow{2}{*}{.142} \\
\hline & Privada & 188 & 160.59 & & & & \\
\hline \multirow{2}{*}{ Desempeño } & Pública & 113 & 146.32 & \multirow{2}{*}{10093.5} & \multirow{2}{*}{-.727} & \multirow{2}{*}{.467} & \multirow{2}{*}{.041} \\
\hline & Privada & 188 & 153.81 & & & & \\
\hline
\end{tabular}

d. Obligaciones de la organización, compromiso con su organización, satisfacción y desempeño laboral, según el estatus ocupacional

La tabla 11, nos muestra que, con respecto al estatus ocupacional, existe diferencias estadísticamente significativas entre las obligaciones de la organización $\left(\chi^{2}=9.534, p<.01\right)$, impactando más en los directivos $(\mathrm{Rp}=198.38)$, seguido por los empleados $(\mathrm{Rp}=147.54)$. Del mismo modo, existe diferencias 
estadísticamente significativas entre satisfacción laboral $\left(\chi^{2}=9.682, p<.01\right)$, según el estatus ocupacional, impactando más en los directivos $(\mathrm{Rp}=197.95)$. Lo que nos muestra, que la percepción de los empleados cambia dependiendo del estatus ocupacional que ostentan, especialmente respecto de obligaciones del empleador y la satisfacción laboral.

\section{Tabla 11}

Obligaciones de la organización, compromiso con su organización, satisfacción y desempeño laboral, según el estatus ocupacional

\begin{tabular}{llllll}
\hline Variable & $\begin{array}{l}\text { Estatus } \\
\text { ocupacional }\end{array}$ & $\boldsymbol{n}$ & $\begin{array}{l}\text { Rango } \\
\text { promedio }\end{array}$ & $\boldsymbol{\chi}^{\mathbf{2}}$ & $\boldsymbol{p}$ \\
\hline \multirow{3}{*}{ Obligaciones } & Empleado & 254 & 147.54 & & \\
& Directivo & 30 & 198.38 & 9.534 & .009 \\
& Otro & 19 & 138.34 & & \\
Compromiso & Empleado & 254 & 148.72 & \multirow{2}{*}{4.863} & .088 \\
& Directivo & 30 & 185.23 & & \\
Satisfacción & Otro & 19 & 143.42 & & .008 \\
& Empleado & 254 & 145.92 & & \\
& Directivo & 30 & 197.95 & & .057 \\
Desempeño & Otro & 19 & 160.79 & & \\
& Empleado & 254 & 147.53 & & \\
& Directivo & 30 & 187.62 & & \\
\hline
\end{tabular}

e. Obligaciones de la organización, compromiso con su organización, satisfacción y desempeño laboral, según la remuneración mensual.

La tabla 12 nos muestra que existen diferencias estadísticamente significativas entre las obligaciones de la organización según la remuneración mensual ( $\mathrm{F}=$ $6.125, p<.01)$, con un tamaño de efecto muy bajo $\left(\omega^{2=} .048\right)$, impactando más entre los que ganan 4001 soles a más $(\mathrm{M}=53.78)$.

Igualmente, existen diferencias estadísticamente significativas entre satisfacción laboral según la remuneración mensual $\left(\chi^{2}=13.089, \mathrm{p}<.01\right)$, impactando más en los que ganan 4001 soles a más $(\mathrm{Rp}=179.40)$. El compromiso de los empleados con su organización y el desempeño laboral no son estadísticamente significativos con la intervención de la remuneración. Es decir, La percepción que tienen los empleados no varía, no se afecta con los ingresos económicos mensuales.

\section{Tabla 12}

Obligaciones de la organización, compromiso con su organización, satisfacción y desempeño laboral, según la remuneración mensual

\begin{tabular}{llllllll}
\hline Variable & Remuneración mensual & $\mathbf{n}$ & $\boldsymbol{M}$ & $\boldsymbol{D E}$ & $\boldsymbol{F}$ & $\boldsymbol{p}$ & $\boldsymbol{\omega}^{2}$ \\
\hline \multirow{3}{*}{ Obligaciones } & 850 a 2000 soles & 84 & 45.80 & 13.79 & & & \\
& 2001 a 3000 soles & 71 & 46.10 & 15.00 & \multirow{2}{*}{6.125} & .000 & .048 \\
& 3001 a 4000 soles & 62 & 47.15 & 13.01 & & & \\
& 4001 soles a más & 86 & 53.78 & 13.53 & & & \\
\hline
\end{tabular}




\begin{tabular}{lllccc}
\hline Variable & Remuneración mensual & $\mathbf{n}$ & $\begin{array}{c}\text { Rango } \\
\text { promedio }\end{array}$ & $\boldsymbol{\chi}^{\mathbf{2}}$ & $\boldsymbol{p}$ \\
\hline \multirow{3}{*}{ Compromiso } & 850 a 2000 soles & 84 & 142.84 & & \\
& 2001 a 3000 soles & 71 & 149.39 & 3.308 & .347 \\
& 3001 a 4000 soles & 62 & 147.99 & & \\
Satisfacción & 4001 soles a más & 86 & 165.99 & & \\
& 850 a 2000 soles & 84 & 134.77 & & \\
& 2001 a 3000 soles & 71 & 150.68 & \multirow{2}{*}{13.089} & .004 \\
& 3001 a 4000 soles & 62 & 138.85 & & \\
Desempeño & 4001 soles a más & 86 & 179.40 & & \\
& 850 a 2000 soles & 84 & 153.49 & & .704 \\
& 2001 a 3000 soles & 71 & 142.36 & \multirow{2}{*}{1.408} & \\
\hline
\end{tabular}

f. Obligaciones de la organización, compromiso con su organización, satisfacción y desempeño laboral, según el lugar de procedencia.

De acuerdo con la tabla 13, existe diferencias estadísticamente significativas entre desempeño laboral según el lugar de procedencia $(Z=-2.458, p<.05)$, impactando más en los trabajadores que proceden de la costa $(R p=154.39)$. Las obligaciones de la organización, el compromiso de los empleados con su organización y la satisfacción laboral no arrojan significancia con la intervención del lugar de procedencia, es decir, el hecho de proceder de una región u otra no cambia la percepción que tienen los empleados.

Tabla 13

Obligaciones de la organización, compromiso con su organización, satisfacción y desempeño laboral, según el lugar de procedencia

\begin{tabular}{llllllll}
\hline Variable & $\begin{array}{l}\text { Lugar de } \\
\text { residencia }\end{array}$ & $\mathbf{n}$ & $\begin{array}{l}\text { Rango } \\
\text { promedio }\end{array}$ & $\boldsymbol{U}$ & $\boldsymbol{Z}$ & $\boldsymbol{p}$ & $\boldsymbol{r}_{p}$ \\
\hline Obligaciones & Costa & 292 & 153.33 & 1218.0 & -1.361 & .174 & .078 \\
& Sierra & 11 & 116.73 & & & & \\
\multirow{2}{*}{ Compromiso } & Costa & 292 & 152.28 & 1525.5 & -.282 & .778 & .016 \\
& Sierra & 11 & 144.68 & & & & \\
\multirow{2}{*}{ Satisfacción } & Costa & 292 & 152.50 & 1459.0 & -.516 & .606 & .029 \\
& Sierra & 11 & 138.64 & & & & \\
\multirow{2}{*}{ Desempeño } & Costa & 292 & 154.39 & 909.0 & -2.458 & .014 & .141 \\
\hline
\end{tabular}

Como se puede apreciar, en los cuadros precedentes, se analizaron también algunas otras variables socio demográficas y organizacionales (edad, grado de instrucción, lugar de residencia, antigüedad en la organización y años de servicio en el puesto de trabajo) y se encontró no existir diferencia estadísticamente significativa con respecto de las obligaciones de la organización, compromisos de los empleados con su organización, satisfacción laboral y desempeño laboral. 
En otros términos, la intervención de las variables socio demográficas y organizacionales indicadas no modifican la percepción de los empleados respecto de las variables principales analizadas.

\section{DISCUSIÓN}

El objetivo de la presente investigación ha sido conocer el estado actual del contrato psicológico y su relación con el desempeño y la satisfacción en empleados del sector público y privado de Lima. A la luz de los resultados se podría afirmar que los empleados perciben que el contrato psicológico en sus dos situaciones (las obligaciones de los empleadores y los compromisos de los empleados con su organización), se encuentran en un nivel medio, similar al encontrado por Gracia, Peiró y Mañas (2007), pero también la satisfacción como el desempeño laboral se encuentran en un nivel medio en este estudio, al igual que indican los hallazgos de Herrera-Amaya y Manrique-Abril (2008); sin embargo, en un análisis comparativo, mientras que el cumplimiento de las obligaciones de las organizaciones y la satisfacción laboral tienden a elevarse, el compromiso de los empleados con su organización y el desempeño laboral tienden a ser más bajos, en la percepción de los propios empleados, tendencia contraria a lo encontrado por Gracia, Peiró y Mañas (2007) y Robbins, (1994), estas evidencias pueden ser el inicio o el anticipo de los riesgos que se presumía podían ocurrir como consecuencia de las nuevas modalidades de empleo y gestión (Gallie \& White,1993), generando un conjunto de consecuencias imprevisibles, pero el más inmediato será la frustración y la ansiedad(Morrison y Robinson, 1997), por tanto, agresiones directas o indirectas, verbales o escritas, actitudes o comportamientos abiertos u ocultos, conscientes o inconscientes.

Un análisis detallado de las relaciones entre las dos situaciones del contrato psicológico, el desempeño y la satisfacción laboral nos indican que cuanto mayor es el cumplimiento de las obligaciones del empleador, mayor será el compromiso de los empleados con su organización, mayor satisfacción laboral y mayor desempeño laboral; del mismo modo, cuanto mayor es el compromiso con su organización, mayor será la satisfacción laboral y mayor el desempeño laboral de los empleados. Así mismo, a mayor satisfacción laboral mayor desempeño laboral, algo similar a lo encontrado por otros investigadores (Topa, Palací y Morales, 2004). Al analizar la ruptura del contrato psicológico (incumplimiento), también encuentran que se asocia con menor confianza, menor compromiso y menos lealtad, sobre todo, se dice que tiene un profundo impacto en el desempeño del trabajo; o los hallazgos de Topa y Morales (2007) que indica que la percepción de ruptura se muestra directamente relacionada con la satisfacción laboral y que la organización como forma de identidad social en el trabajo predice mejor la satisfacción con factores higiénicos; contrario a lo encontrado por Ferro y Gómez (2014) donde la satisfacción laboral no está asociada al contrato psicológico. 
Este último hallazgo, nos puede estar indicando el viraje de las expectativas y sus efectos de la nueva relación laboral. Pero, por ahora, la mayoría de los estudios sobre incumplimiento del contrato psicológico confirman nuestra hipótesis, afectando, sin lugar a dudas, la satisfacción y el desempeño laboral, como bien señalan los hallazgos de Rosario-Hernández y Rovira (2006). El compromiso de los empleados hacia su organización, como la satisfacción y el desempeño laboral son gestos de compensación y reconocimiento al cumplimiento de las obligaciones implícitas sostenibles y las buenas prácticas de gestión, como se confirma en estudios realizados por Silla, Gracia y Peiró (2014).

Por otro lado, analizando las mismas variables y los cambios que puedan operarse, dependiendo de las condiciones sociodemográficas, se encontró diferencias de percepción de acuerdo con el género, el estado civil, el tipo de organización, el estatus ocupacional, la remuneración y la procedencia. Es decir, cuando intervienen estas últimas variables, la percepción sobre las obligaciones de las organizaciones, el compromiso de los empleados con su organización, y la percepción sobre su satisfacción laboral y su desempeño laboral varían, no son homogéneos. Entre tanto, no se dan estas variaciones en la percepción de los empleados cuando interviene la edad, el grado de instrucción, lugar de residencia y antigüedad en el puesto y en la organización del sector público como privado de Lima. Situación ésta, contraria a lo encontrado por Böhrt, Solares y Romero (2014) donde a mayor edad, se da mayor grado de compromiso; respecto de la antigüedad, a medida que pasa el tiempo el contrato psicológico se deteriora y la capacidad de respuesta de los trabajadores es menor.

Como se puede ver, hay algunos aspectos que aún están para seguir explorando, tal vez se debe al hecho de que los participantes de las muestras de estudio pertenecen a diversas culturas, pero también puede ser por los cambios en las relaciones laborales mismas, en la medida que la cultura que trae consigo la globalización, las expectativas económicas desmesuradas y su prevalencia superflua estén socavando las bases morales, la ética y otros valores más preciados del hombre y la sociedad.

\section{CONCLUSIONES}

1. El nivel de cumplimiento de obligaciones de la organización y la satisfacción laboral, respecto del contrato psicológico, en la percepción de la mayoría de los empleados de las organizaciones públicas y privadas de Lima, se encuentran en el nivel medio, con ligera tendencia a elevarse.

2. El nivel de compromiso de los empleados con su organización y el desempeño laboral, respecto del contrato psicológico, en la percepción de la mayoría de los empleados de las organizaciones públicas y privadas de Lima, se encuentran en el nivel medio, con ligera tendencia a bajar. 
3. Existe asociación entre las obligaciones del empleador, el compromiso de los empleados con su organización, la satisfacción laboral y el desempeño laboral. De manera que, a mayor cumplimiento de las obligaciones del empleador, habrá mayor compromiso de los empleados con su organización, y también mayor satisfacción laboral y mayor desempeño laboral.

4. Existe diferencias significativas entre las obligaciones del empleador, el compromiso de los empleados con su organización, la satisfacción laboral y el desempeño laboral con algunas variables sociodemográficas (sexo, estado civil, estatus ocupacional, remuneración y procedencia). Significa que, al intervenir estas variables, la opinión de los empleados cambia respecto de las obligaciones del empleador, el compromiso de los empleados con su organización, la satisfacción laboral y el desempeño laboral.

5. Existe diferencias significativas entre las obligaciones de la organización y la satisfacción laboral según el tipo de organización, siendo más alto en las organizaciones privadas que públicas. Significa, que dependiendo del tipo de organización en la cual trabajan los empleados (pública o privada), la percepción de los mismos cambia respecto de las obligaciones de la organización, así como de la satisfacción laboral.

6. No existe diferencia significativa alguna entre las obligaciones del empleador, el compromiso de los empleados con su organización, la satisfacción laboral y el desempeño laboral según la edad, grado de instrucción, lugar de residencia, años de servicio en el puesto y en la organización; es decir, un análisis con estas variables nos permitió saber que la opinión de los participantes de la muestra no cambia.

\section{RECOMENDACIONES}

1. Las organizaciones públicas como las privadas, deben considerar dentro de sus políticas de gestión humana las obligaciones y los compromisos implícitos adquiridos en la relación laboral, si existe el deseo de mantener satisfecho a sus empleados y que tengan un alto desempeño.

2. Las organizaciones tienen la potestad de seleccionar a las personas en virtud de sus competencias y contratarlas a aquellas que mejor les parece, pagarle una remuneración justa por las actividades que debe desarrollar, y completar, si es necesario, la formación de sus competencias, a fin de potenciar al máximo sus capacidades para un alto desempeño. Estas características pueden formar parte del contrato formal, sin embargo, lo que deben preocuparse también es acerca de brindarle un clima social y organizacional saludable y seguro, oportunidades de desarrollo profesional y personal, posibilidades de promoción y ascenso; vale 
decir, crecimiento psicológico de su capital humano. Este, que no tuvo oportunidad de expresar ni poner condiciones respecto de sus expectativas durante la formalización del contrato.

3. En relación con lo anterior, deberá gestionar el clima social y organizacional y realizar programas de motivación, talleres de desarrollo personal y de capacitación acorde con las tareas del puesto de trabajo, propiciar relaciones saludables con los jefes, oportunidades de cooperación y trabajo en equipo, otorgar libertad y autonomía para la toma de decisiones respecto de su trabajo, brindarle un trato justo y respetuoso, comunicación abierta, beneficios sociales, entre otros, de suerte que cubra las expectativas del trabajador.

\section{REFERENCIAS}

Blanch, J. y Cantera, L. (2009). El malestar en el empleo temporal involuntario. Revista de Psicología del Trabajo y de las Organizaciones, 25(1), 59-70. Recuperado desde: http://www.redalyc.org/pdf/2313/231316499007.pdf

Böhrt, R.; Solares, L. y Romero, C. (2014). Evolución del contrato psicológico y el compromiso organizacional con la edad y la antigüedad. Rev. Ajayu, 12(1), 33-63.

Calderón, G.; Naranjo, J. y Álvarez, C. (2010). Gestión humana en la empresa colombiana: sus características, retos y aportes. Una aproximación a un sistema integral. Cuadernos de Administración, 23(41),13-36.

De Luca, M. P. (2012). El contrato psicológico en empresas familiares: diferencias en su contenido, cumplimiento e incumplimiento respecto a las no familiares. Revista de Psicología GEPU, 3(2), .89-125.

Dunahee, M. \& Wangler, L. (1974). The psychological contract: A conceptual structure for management/employee relations. Personnel Journal, 518-526.

Ferro, J. y Gómez, G. (2014). Relación entre la satisfacción laboral, el contrato psicológico, el tipo de vinculación y la antigüedad en docentes de una universidad privada. Global Conference on Business and Finance Proceedings 9(2). Recuperado desde: ftp://ftp. repec.org/opt/ReDIF/RePEc/ibf/riafin/riaf-v8n7.../RIAF-V8N7-2015-4.pdf

Gallie, D. \& White, M. (1993) Employee Commitment and the Skills Revolution, London: PSI Publishing

Gracia, F.; Silla, I.; Peiró, J. y Fortes-Ferreira, L. (2006). El estado del contrato psicológico y su relación con la salud psicológica de los empleados. Psicothema, 18(2), 256-262.

Gracia, F.; Peiró, J. y Mañas, M. (2007). El contrato psicológico en la Administración Pública: derechos y obligaciones del empleado público, desde su propia perspectiva. Rev. de Psicología del Trabajo y de las Organizaciones, 23(3), 389-418.

Herrera-Amaya, G. y Manrique-Abril, F. (2008). Condiciones laborales y grado de satisfacción de profesionales de enfermería. Colombia: Rev. Aquichan, 8(2), 243-256. 
Hurst, J.; Good, L. \& Gardner, P. (2012). Conversion intentions of interns: what are the motivating factors. Education Training 54 (6), 504-522. Recuperado desde: http:// www.emeraldinsight.com/doi/pdfplus/10.1108/00400911211254280

Huse y Bowditch (1988), El comportamiento humano en la organización. Bilbao: Deusto.

Levinson, H. (1962). Men, management, and mental helth. Cambridge, Mass: Harvard University Pres.

Morrison, E. \& Robinson, S. (1997). When employees feel betrayed: A model of how psychological contract violation develops. Academy of Management Review, 22 (I): 226-256.

Robbins, S. (1994). Comportamiento organizacional. México: Prentice Hall International.

Rosario-Hernández, E. y Rovira, L. (2006). Posibles predictores de conductas de ciudadanía organizacional en una muestra de empleados de Puerto Rico. Rev. Interamericana de Psicología Ocupacional, 25(2), 71-86. Recuperado desde:http:// revista.cincel.com.co/cincel/index.php/RPO/article/view/69

Rousseau, D. (1989). Psychological and impliedcontracts in organizations. EmployeeResponsibilities and RightsJournal, 2, 121-139.

Saldarriaga, L. (2013). El contrato psicológico en las organizaciones, un fenómeno real. Rev. Poiésis, 26. Recuperado desde: http://www.funlam.edu.co/revistas/index.php/ poiesis/article/view/1018

Schein, E.H. (1982). Psicología de la organización. Colombia: Prentice-Hall Internacional.

Silla, I.; Gracia, F. y Peiró, J. (2005). Diferencias en el contenido del contrato psicológico en función del tipo de contrato y de la gestión empresarial pública o privada. Rev. Psicología Social, 20(1), 61-72. Recuperado desde: https:/dialnet.unirioja.es/servlet/ articulo?codigo $=1068806$

Solanes, A. y Zaragoza, B. (2010). ¿Trabajo de preferencia o contrato indefinido?: Un análisis de su impacto sobre el estado del contratopsicológico. Rev. Acciones e Investigaciones Sociales, 28, 57-77.

Tena, G. (2002).El contrato psicológico: relación laboral empresa - trabajador. Acciones e Investigaciones Sociales, 15, 85-107. Recuperado desde: https://dialnet.unirioja.es/ descarga/articulo/284117.pdf

Topa, G. y Morales, J. (2007). Identificación organizacional y ruptura de contrato psicológico: sus influencias sobre la satisfacción de los empleados. International Journal of Psychology and Psychological Therapy, 7(3), 365-379. Recuperado desde: www.redalyc.org/pdf/560/56070305.pdf

Topa, G. y Palací, F. (2004). ¿Ruptura o cumplimiento del contrato psicológico? una revisión meta-analítica de la investigación empírica. Rev. Acción psicológica, 3, 155-171.

Topa, G.; Palací, F. y Morales, J. (2004). La ruptura de contrato psicológico y las respuestas del trabajador. ¿Relaciones mediadas por la confianza organizacional? Rev. Psicología del Trabajo y de las Organizaciones, 20(1), 31-45. 